\title{
Light quark masses from Domain Wall Fermions
}

\author{
C. Dawson ${ }^{a}[\text { RBC Collaboration }]^{*}$ \\ ${ }^{a}$ RIKEN-BNL Research Center,Bldg 510a, Upton, NY 11973-5000
}

\begin{abstract}
We present results for the renormalised light and strange quark masses calculated using Domain Wall Fermions in quenched QCD. New results using the DBW2 gauge action at $a^{-1} \approx 2 \mathrm{GeV}$ and $a^{-1} \approx 1.3 \mathrm{GeV}$ will be presented and compared against existing results at $a^{-1} \approx 2 G e V$ using the Wilson gauge action. This comparison allows a study of the uncertainties due to both finite lattice spacing and residual chiral symmetry breaking effects.
\end{abstract}

\section{INTRODUCTION}

We report here results for the light and strange quark masses extracted from the quark mass dependence of the meson spectrum calculated using domain wall fermions [1]2] (DWF), see [3] for our notation and conventions, in the quenched approximation and renormalised using the nonperturbative renormalisation (NPR) technique developed by the Rome-Southampton group [4].

All the results presented will be from simulation using a lattice with spatial dimensions $16^{3} \times 32$ and a fifth dimension of size 16 . The new results use the DBW2 gauge action [5] at $\beta=0.87$ and $\beta=1.04$ corresponding to $a^{-1} \approx 1.3 \mathrm{GeV}$ and $a^{-1} \approx 2 \mathrm{GeV}$ respectively. This action is chosen for its much smaller explicit chiral symmetry breaking when compared to other actions $[6]$. The results will be compared with those of previous studies using the Wilson gauge action with $\beta=6.0$ [3, 7, 8 ]. For $\beta=0.87, M_{5}=1.8$ was used and 53 and 100 configurations were collected for the NPR and hadron spectrum respectively, whereas for $\beta=1.04, M_{5}=1.7$ was used and 51 and 405 configurations were collected.

\section{RENORMALISATION}

Our final result will be the renormalised mass in the $\overline{M S}$-scheme at $2 \mathrm{GeV}$. The relation between this value and the bare mass, $m_{f}$, consists

\footnotetext{
${ }^{*}$ We thank RIKEN, Brookhaven National Laboratory and the U.S. Department of Energy for providing the facilities essential for the completion of this work.
}

of several factors and may be written

$m_{\text {ren }}=Z^{\text {match }} Z_{m}^{R I}\left(m_{f}+m_{\text {res }}\right)$.

The residual mass, $m_{\text {res }}$, occurs because of small explicit chiral symmetry breaking effects due to the finite extent of the fifth dimension. This we determined from the breaking term of the axial Ward-Takahashi identity [3]. $Z_{m}^{R I}$ is the mass renormalisation factor in the RI/MOM-scheme which is calculated directly on the lattice using the method of the Rome-Southampton group [4] and then matched to the $\overline{M S}$-scheme at $2 \mathrm{GeV}$ using a two-loop perturbative matching factor, $Z^{\text {match }}$ [9].

For the NPR technique to be successful a "window" of momenta must exist for which contamination due to (low-momenta) condensate effects are small but also for which (high-momenta) lattice artifacts are suppressed. Previous work shows that when using DWF with the Wilson action at $a^{-1} \approx 2 \mathrm{GeV}$ this window exists 8 . In this work we confirmed that this is also the case for the DBW2 action and checked the feasibility of NPR at $a^{-1} \approx 1.3 \mathrm{GeV}$.

While $Z_{m}$ may be calculated directly from the momentum-space quark propagator this approach has large systematic errors. Instead we consider the renormalisation of the flavour nonsinglet local quark bilinear operator, $\bar{u} \Gamma d$. The NPR technique provides a clean extraction of the ratio $Z_{\Gamma} / Z_{q}$, where $Z_{q}$ is the quark renormalisation factor. Putting the value of $Z_{S} / Z_{q}$ (scalar) and $Z_{A} / Z_{q}$ (axial) together with the value of $Z_{A}$ calculated from the hadronic matrix element of 


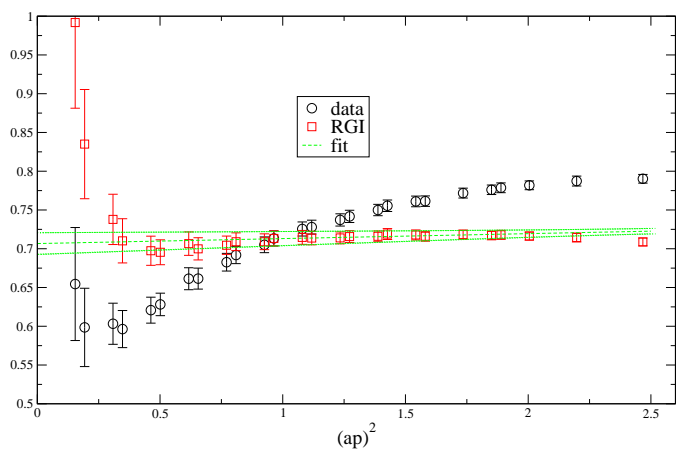

Figure 1. $Z_{S} / Z_{q}$ bare and RGI for the DBW2 action at $\beta=1.04$

the local and conserved axial currents with the pseudo-scalar density 3] gives our final value for $Z_{S}$. This can then be used to calculate $Z_{m}$ as $Z_{S}=1 / Z_{m}$.

An important check of the existence of a "window" may be made by dividing $Z_{S} / Z_{q}$ by the predicted running from 3-loop perturbation theory to produce a renormalisation group invariant (RGI) value. Fig. 11 shows this for $\beta=1.04$ DBW2 where the RGI and bare data are constrained to agree at $(a p)^{2}=1$.

Fig. 2 show this for $\beta=0.87$ DBW2 (in this case the bare and RGI are constrained to agree at $\left.(a p)^{2}=1.5\right)$. There is both more evidence of curvature at low-momenta due to condensate effects and a noticeable slope versus $(a p)^{2}$ at high momenta. Interpreting this slope as $O\left(a^{2}\right)$ contamination and fitting the RGI value to $A+B(a p)^{2}$ allows the final value for $Z_{S} / Z_{q}$ to be extracted.

Table 1 collects together the (preliminary) DBW2 results for $Z_{A}$ and $Z_{S} / Z_{A}$ (the latter in the $\overline{M S}$-scheme at $2 \mathrm{GeV}$ ), and the results for Wilson gauge results already quoted in [8].

Table 1

Results for $Z_{A}$ and $Z_{S} / Z_{A}$

\begin{tabular}{cccc}
\hline Action & $\beta$ & $Z_{A}$ & $Z_{S} / Z_{A}$ \\
\hline DBW2 & 0.87 & $0.7776(5)$ & $0.898(16)(90)$ \\
DBW2 & 1.04 & $0.8402(2)$ & $0.849(15)(40)$ \\
Wilson & 6.00 & $0.7555(3)$ & $0.830(09)(35)$ \\
\hline
\end{tabular}

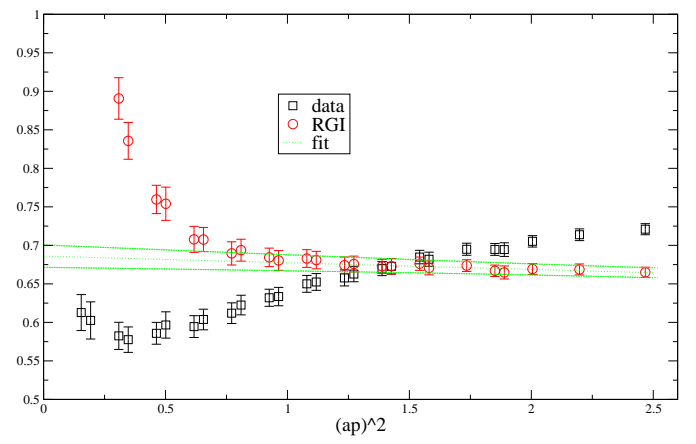

Figure 2. $Z_{S} / Z_{q}$ bare and RGI for the DBW2 action at $\beta=0.87$

\section{MESON MASSES}

For the pseudo-scalar meson masses chiral perturbation theory to first order tells us that

$m_{\pi}^{2} a^{2}=B_{\pi} a \bar{m}$

$m_{K}^{2} a^{2}=B_{\pi} a\left(m_{s}+\bar{m}\right) / 2$

where $\bar{m}=\frac{1}{2}\left(m_{u}+m_{d}\right)$, while for the vector mesons the leading dependence should be linear in the quark masses

$m_{\rho} a=A_{\rho}+B_{\rho} \bar{m}$.

Our approach to calculating the quark masses is to first fit the meson spectrum for the coefficients $B_{\pi}, A_{\rho}$ and $B_{\rho}$ and then to use the physical values for $m_{\pi}, m_{K}$ and $m_{\rho}$ to calculate $a, \bar{m}$ and $m_{s}$.

In extracting the meson masses we must deal with two systematic effects due to working in the quenched approximation: the contamination of meson correlators due to the presence of unsuppressed topological near zero-modes [3], and significant deviations from Eq. 22 at light masses due to quenching effects.

The effects of zero-modes are most apparent at light masses and small volumes, but also are strongly dependent on which correlators are used to calculate the mass. In particular when extracting the pseudo-scalar meson mass the zeromode effects may be reduced (but not completely avoided) by calculating the mass from axial correlators rather than the more usual pseudo-scalar correlators. In addition to using the axial correlator for the pseudo-scalar mass extraction, we 


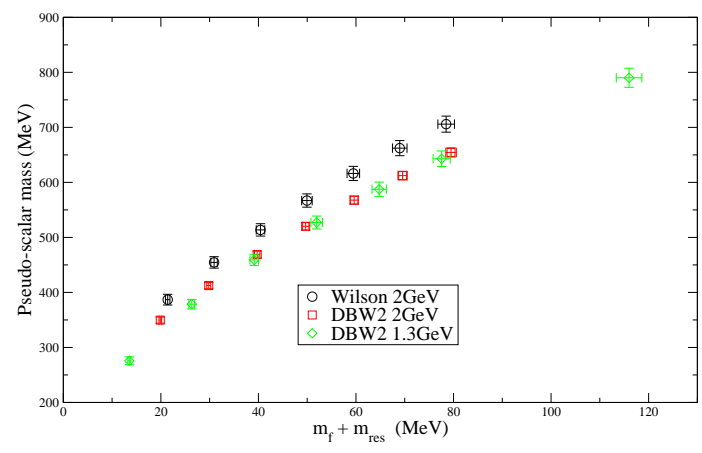

Figure 3. Pseudo-scalar mass versus $m_{f}+m_{\text {res }}$

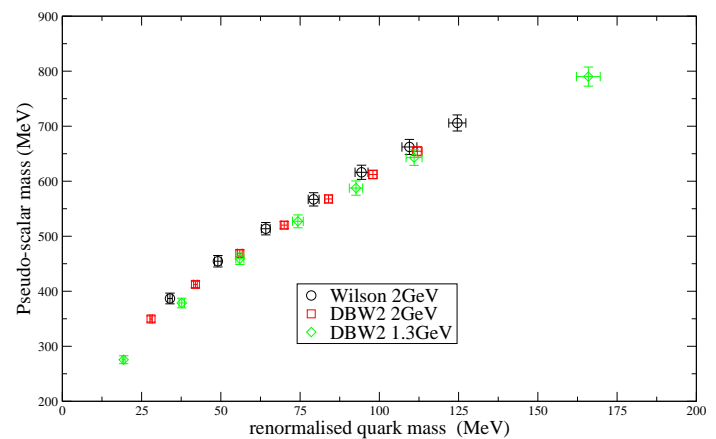

Figure 4. Pseudo-scalar mass versus renormalised mass

only use bare masses greater than $m_{f}=0.01$ in our analysis.

Using the mass extracted from the axial correlator, the consistency of our different data-sets with each other and the NPR calculation may be tested. Fig. 3 shows the pseudo-scalar mass versus $\tilde{m}=m_{f}+m_{\text {res }}$, both measured in $\mathrm{MeV}$, whereas Fig. 1 shows the same data versus renormalised mass. As can be seen once the renormalisation factors are taken into account the data-sets show excellent consistency both between the two gauge action at the same lattice spacing and the two different lattice spacing with the same gauge action. The deviation from Eq. 2 2 is very apparent in our data with a simple linear fit of

$\left(a m_{\pi}\right)^{2}=A_{\pi}+B_{\pi} a \tilde{m}$

for the $\beta=1.04$ DBW2 data having a constant term, $A_{\pi}$, inconsistent with zero by 3 standard deviation whereas the intercept of a more general quadratic fit misses zero by 7 standard deviations.

As this is the case we include a quenched chiral logarithm in our fitting formula (but drop its effects for the final calculation of the quark masses).

$$
\left(a m_{\pi}\right)^{2}=B_{\pi} \tilde{m}\left(1-\delta \log \left(\frac{B_{\pi} \tilde{m}}{\Lambda^{2}}\right)\right)
$$

where we use a chiral scale of $\Lambda=1 \mathrm{GeV}$ [10]. This gives very consistent values of $\delta=5.1(19) \times$ $10^{-2}$ and $\delta=4.6(18) \times 10^{-2}$ for Wilson and DBW2 at $2 \mathrm{GeV}$ and $\delta=2.28(12) \times 10^{-2}$ at $1.3 \mathrm{GeV}$. However, none of the fits are of high quality and the best treatment of the quenching effects is an issue that requires further study.

\section{RESULTS}

Putting together the NPR results and the fits to the meson spectrum gives values for the quark masses in $\overline{M S}$-scheme at $2 \mathrm{GeV}$. Using Equations 2 to 1 implies that

$m_{s} / \bar{m}=2 m_{K}^{2} / m_{\pi}^{2}-1 \approx 26$

exactly for our results so we only quote the strange quark mass. For this we get 132.5(31) $\mathrm{MeV}(\beta=0.87$, DBW2), $132.6(21) \mathrm{MeV}(\beta=$ 1.04 , DBW2), and $m_{s}=125.6(28) \mathrm{MeV}(\beta=6.0$, Wilson) where the errors quoted are purely statistical.

\section{REFERENCES}

1. D. B. Kaplan, Phys. Lett. B288 (1992) 342

2. V. Furman and Y. Shamir, NPB439 (1995) 54

3. T. Blum et al., (2000), hep-lat/0007038.

4. G. Martinelli et al., NPB445 (1995) 81

5. QCD-TARO, P. de Forcrand et al., NPB577 (2000) 263

6. RBC, K. Orginos, Nucl. Phys. Proc. Suppl. 106 (2002) 721

7. T. Blum, A. Soni and M. Wingate, PRD60 (1999) 114507

8. T. Blum et al., PRD66 (2002) 014504

9. K.G. Chetyrkin and A. Retey, NPB583 (2000) 3

10. T. Blum et al., (2001), hep-lat/0110075. 